\title{
Multiple Zeros for Eigenvalues and the Multiplicity of Traps of a Linear Compartmental System
}

\author{
DAVID M. FOSTER*
}

AND

JOHN A. JACQUEZ

University of Michigan, Department of Physiology,

7712 Medical Science II, Ann Arbor, Michigan 48104

\section{ABSTRACT}

It is known that a linear compartmental system has a trap if and only if the associated system of differential equations has zero as an eigenvalue. In this paper, we show that if such a system has zero as an eigenvalue of multiplicity $m$, then the system contains $m$ irreducible traps.

In [1], Fife showed that a linear compartmental system has a trap if and only if the associated system of differential equations has zero as an eigenvalue. The question arises as to what information about the system can be gained from knowing the multiplicity of this eigenvalue. In this note, we will use some results given by Hearon [2] to examine this question.

Let $S$ be a linear compartmental system consisting of $n$ compartments $C_{1}, \ldots, C_{n}$, and let $q_{j}$ be the amount of material in $C_{j}$. Let $f_{i j}$ be the fractional exchange coefficient, so that the rate of flow of material from $C_{j}$ to $C_{i}$ is $f_{i j} q_{j}$. Let $f_{0 j} q_{j}$ be the rate of flow of material from $C_{j}$ to the environment. Setting

$$
f_{j j}=-\left(f_{0 j}+\sum_{\substack{i=1 \\ i \neq j}}^{n} f_{i j}\right)
$$

*This work was done while the senior author was a post-doctoral fellow supported by NIH Research Fellowship GM00110. 
we observe that $f_{j} q_{j}$ is the total amount of material leaving $C_{j}$ per unit time. Therefore, the rate of change of material in $C_{j}$ is given by

$$
\frac{d q_{j}}{d t}=f_{j j} q_{j}+\sum_{\substack{i=1 \\ i \neq j}}^{n} f_{j i} q_{i}, \quad i=1, \ldots, n .
$$

In matrix notation, (2) becomes

$$
\dot{q}=F q,
$$

where $q=\left(q_{1}, \ldots, q_{n}\right)^{T}$ and $F=\left(f_{i j}\right)$. We call $\left(f_{i j}\right)$ the matrix of the system (relative to $\left\{C_{1}, \ldots, C_{n}\right\}$ ).

The system $S$ is called a separable linear system if it can be partitioned into a disjoint union of subsystems

$$
S=S_{1} \dot{\cup} S_{2} \dot{U} \cdots \dot{\text { i }} S_{k}
$$

such that $S_{i}$ receives no input from $S_{i+1}, \ldots, S_{k}, i=1, \ldots, k-1$. Suppose $S$ is separable. Then it is clear that we may renumber the compartments of $S$ so that

$$
\begin{aligned}
& S_{1}=\left\{C_{1}, \ldots, C_{k_{1}}\right\}, \\
& S_{2}=\left\{C_{k_{1}+1}, \ldots, C_{k_{2}}\right\}, \\
& \vdots \\
& S_{k}=\left\{C_{k_{k-1}}, \ldots, C_{n}\right\},
\end{aligned}
$$

where, relative to this numbering, $F$ assumes block triangular form

$$
F=\left[\begin{array}{llll}
F_{11} & & & \\
F_{21} & F_{22} & 0 & \\
\vdots & \vdots & \ddots & \\
F_{k 1} & F_{k 2} & \ldots & F_{k k}
\end{array}\right]
$$

Now let $\Sigma_{n}$ be the permutation group on $\{1,2, \ldots, n\}$. Then an arbitrary $n$ by $n$ matrix $A=\left(a_{i j}\right)$ is, by definition, reducible iff there exists a $\sigma$ in $\Sigma_{n}$ such that

$$
\left(a_{\boldsymbol{\sigma}(i), \boldsymbol{\sigma}(j)}\right)=\left[\begin{array}{cc}
A_{11} & 0 \\
A_{21} & A_{22}
\end{array}\right],
$$

where $A_{11}$ and $A_{22}$ are square matrices of dimension less than $n$ [2]. 
Consequently, if $\sigma \in \Sigma_{n}$, then $\left(f_{\sigma(i), \sigma(j)}\right)$ is the matrix of $S$ relative to $\left\{C_{\sigma(1)}, \ldots, C_{\sigma(n)}\right\}$. It follows easily that if $F$ is reducible, then $S$ is separable, and conversely.

Let us consider (4). We recall that a subsystem $S_{m}$ is a trap iff it has no output to the rest of the system or to the environment. Thus, if $S_{m}$ is a trap, $F_{i m}=0$ for $i=1, \ldots, k, i \neq m$, and, according to Fife [1], $\operatorname{det}\left(F_{m m}\right)=0$.

Now suppose $S$ is not separable and open (in the sense that $f_{0 i} \neq 0$ for at least one $i$ ). Then $F$ is irreducible and, for at least one $i$,

$$
\left|f_{i i}\right|>\sum_{\substack{j=1 \\ i \neq j}}^{n} f_{j i}
$$

In this situation, $F$ is non-singular (see Taussky [3]). On the other hand, if $S$ is closed, then zero is a simple eigenvalue of $F$ (see Hearon [2], p. 45). We note that adding an excretion from any compartment of $S$ to the environment makes $S$ open, but still leaves $F$ irreducible. Thus the system as a trap can have the trap removed by adding an excretion from any compartment.

Now suppose $S$ is separable, and zero is a simple eigenvalue of $F$. Then by an easy argument (see Hearon [2]), $S$ can be expressed as a disjoint union of two subsystems

$$
S=T_{1} \cup T_{2}
$$

where $T_{2}$ is a trap and, as a system, is not separable. The matrix of this system assumes the form

$$
F=\left[\begin{array}{cc}
G_{11} & 0 \\
G_{21} & G_{22}
\end{array}\right]
$$

where $G_{11}$ is non-singular and $G_{22}$ has zero as a simple eigenvalue and is irreducible. As above, adding an excretion from any compartment in $T_{2}$ will remove $T_{2}$ as a trap.

We are now ready to give

\section{THEOREM}

Suppose $S$ is a linear compartmental system, and suppose zero is an eigenvalue of multiplicity $m$ of the system matrix.

1. If $m=1$, then $S$ is separable iff the system matrix is reducible.

2. If $m>1$, the system $S$ is separable. 
3. There exists a partitioning of $S$ into a disjoint union of subsystems

$$
S=S_{1} \dot{\cup} S_{2} \dot{U} \cdots \dot{\cup} S_{k}
$$

such that $S_{i}$ receives no input from $S_{i+1}, \ldots, S_{k}, i=1, \ldots, k-m$, and $S_{k-m+1}, \ldots, S_{k}$ are traps.

4. Relative to this partitioning (renumbering the compartments of $S$ if necessary), the system matrix is given by

$$
F=\left[\begin{array}{cccccc}
F_{11} & & & & & \\
F_{21} & F_{22} & & & & \\
\vdots & \vdots & \ddots & & 0 & \\
F_{k-m+1,1} & F_{k-m+1,2} & \ldots & F_{k-m+1, k-m+1} & & \\
\vdots & \vdots & & & \ddots & F_{k k} \\
F_{k 1} & F_{k 2} & \cdots & 0 & \cdots &
\end{array}\right]
$$

where, for $i=k-m+1, \ldots, k$, zero is a simple eigenvalue of $F_{i i}$.

5. The addition of an excretion from any compartment of $S_{i}, i=k-m+$ $1, \ldots, k$ removes $S_{i}$ as a trap.

Proof. The proof is by induction on $m$, the case when $m=1$ being proved in the remarks preceding the statement of the theorem. Moreover, if $m>1$, it is clear that $S$ is separable.

Now let us assume that the theorem is valid for all systems whose system matrices have zero as an eigenvalue of multiplicity $m$, and suppose $S$ is an $n$ compartmental system whose system matrix has zero as an eigenvalue of multiplicity $m+1$. Then $S$ is separable, whence

$$
S=T_{1} \dot{\cup} T_{2}
$$

where $T_{1}$ receives no input from $T_{2}$. Renumbering the compartments of $S$ if necessary, the system matrix $F$ assumes the form

$$
F=\left[\begin{array}{cc}
G_{11} & 0 \\
G_{21} & G_{22}
\end{array}\right]
$$

where $G_{11}=\left(g_{i j}\right), i, j=1, \ldots, p ; G_{22}=\left(g_{i j}\right), i, j=p+1, \ldots, n ;$ and $G_{21}=\left(g_{i j}\right)$, 
$i=p+1, \ldots, n, j=1, \ldots, p$. Notice that

$$
g_{j j}=\left\{\begin{array}{l}
-\left(g_{0 j}+\sum_{\substack{i=1 \\
i \neq j}}^{n} g_{i j}\right), \\
-\left(g_{0 j}+\sum_{\substack{i=p+1 \\
i \neq j}}^{n} g_{i j}\right), \quad j=1, \ldots, p
\end{array}\right.
$$

We now make two critical observations. First, let us write

$$
\begin{aligned}
g_{j j} & =-\left[\left(g_{0 j}+\sum_{i=p+1}^{n} g_{i j}\right)+\sum_{\substack{i=1 \\
i \neq j}}^{p} g_{i j}\right] \\
& =g_{0 j}^{\prime}+\sum_{\substack{i=1 \\
i \neq j}}^{p} g_{i j}, \quad j=1, \ldots, p .
\end{aligned}
$$

If we do this, we see that we may regard $G_{11}$ as the system matrix of $T_{1}$, considering $T_{1}$ as a separate system. Notice how we have lumped excretions into $T_{2}$ as "environmental" to accomplish this. It is easy to see how $G_{22}$ may be regarded as the system matrix of $T_{2}$, considering $T_{2}$ as a separate system.

The second observation involves what happens when we apply row and column operations to $F$. Specifically, if we consider $\Sigma_{p}$ as a subgroup of $\Sigma_{n}$, then for any $\sigma \in \Sigma_{p} \subset \Sigma_{n}$, $\left(g_{\sigma(i), \sigma(j)}\right)$ is still block triangular. The effect of putting $G_{11}$ into block triangular form by this method simply rearranges the columns of $G_{21}$. Similarly if $\Sigma_{n-p}^{*}$ is the permutation group on $\{p+1, \ldots, n\}$ and is identified in the natural way as a subgroup of $\Sigma_{n}$, then the effect of putting $G_{22}$ into block triangular form by this method is simply to alter the rows of $G_{21}$.

From these two observations, the proof is immediate. Indeed, without loss of generality, we may assume $\operatorname{det}\left(G_{11}\right)=\operatorname{det}\left(G_{22}\right)=0$. By the induction hypothesis $T_{1}$ and $T_{2}$ can be arranged according to the statement of the theorem, i.e.

$$
\begin{aligned}
& T_{1}=T_{11} \dot{\cup} \cdots \dot{\cup} T_{1 r} \dot{\cup} \cdots \dot{U} T_{1 s}, \\
& T_{2}=T_{21} \dot{\cup} \cdots \dot{\cup} T_{2 u} \dot{\cup} \cdots \dot{U} T_{2 v},
\end{aligned}
$$

where $T_{1 r+1}, \ldots, T_{1 s}, T_{2 u+1}, \ldots, T_{2 v}$ are the $m+1$ traps. Since traps have no excretions, the final arrangement is obvious. (Notice that this is true of the whole matrix, since the column entries under a trap are zeros). Q.E.D. 
We wish to consider two examples to illustrate what the theorem will and won't do.

Example 1. Consider a four compartment system $S=\left\{C_{1}, C_{2}, C_{3}, C_{4}\right\}$ whose connectivity diagram is given by

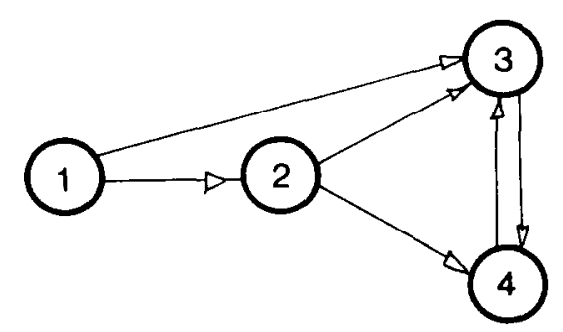

The system matrix according to this numbering is given by

$$
F=\left[\begin{array}{cccc}
f_{11} & 0 & 0 & 0 \\
f_{21} & f_{22} & 0 & 0 \\
f_{31} & f_{32} & f_{33} & f_{34} \\
0 & f_{42} & f_{43} & f_{44}
\end{array}\right]
$$

According to the theorem, the block triangular form of $F$ is

$$
F=\left[\begin{array}{ccc}
F_{11} & 0 & 0 \\
F_{21} & F_{22} & 0 \\
F_{31} & F_{32} & F_{33}
\end{array}\right],
$$

where $F_{11}=\left(f_{11}\right), F_{22}=\left(f_{22}\right)$, and $F_{33}=\left[\begin{array}{ll}f_{33} & f_{34} \\ f_{43} & f_{44}\end{array}\right]$, and zero is a simple eigenvalue of $F_{33}$. Notice this decomposition is relative to

$$
S=S_{1} \dot{\cup} S_{2} \dot{\cup} S_{3}
$$


where $S_{1}=\left\{C_{1}\right\}, S_{2}=\left\{C_{2}\right\}$, and $S_{3}=\left\{C_{3}, C_{4}\right\}$. Now $S_{3}$ is a trap. But $S_{2} \dot{\cup} S_{3}$ is also a subsystem which is a trap, and the whole system, being closed, is a trap. Thus how many traps are there in this system? In light of this, what the theorem tells us directly is the number of irreducible subsystems (subsystems which, when regarded as systems, are not separable) which are traps. Indeed, this is the multiplicity of zero as an eigenvalue. In this example, $S_{3}$ is such a system. What the theorem tells us indirectly is how these traps will nest with other subsystems to form more complex traps. From the form for $F$ given in the theorem, we may draw a connectivity diagram for the $S_{i}$, for $S_{i}$ receives from $S_{j}$ iff $F_{i j} \neq 0$. In this way, we see how complex subsystems which are traps in themselves may be formed. In this particular example,

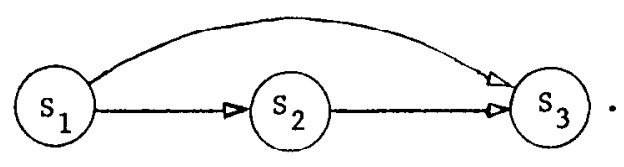

Notice that even though $S_{3}$ is a trap, $S_{1} \dot{\cup} S_{3}$ is not a trap.

Example 2. Consider a nine compartment system $\left\{C_{1}, \ldots, C_{9}\right\}$ whose matrix is given:

$$
\left(f_{i j}\right)=\left[\begin{array}{ccccccccc}
f_{11} & 0 & f_{13} & 0 & 0 & 0 & 0 & 0 & 0 \\
f_{21} & f_{22} & 0 & f_{24} & 0 & 0 & 0 & 0 & 0 \\
0 & 0 & f_{33} & 0 & 0 & 0 & 0 & f_{38} & 0 \\
0 & f_{42} & f_{43} & f_{44} & 0 & 0 & 0 & 0 & 0 \\
0 & 0 & f_{53} & 0 & f_{55} & 0 & 0 & f_{58} & 0 \\
0 & 0 & 0 & 0 & 0 & 0 & 0 & f_{68} & f_{69} \\
0 & 0 & 0 & 0 & f_{75} & 0 & f_{77} & f_{78} & 0 \\
0 & 0 & 0 & 0 & f_{85} & 0 & 0 & f_{88} & 0 \\
0 & 0 & f_{93} & 0 & 0 & 0 & 0 & 0 & f_{99}
\end{array}\right]
$$

where $f_{0 i}=0$ except for $i=7$. From the matrix, we can read off the 
connectivity diagram:

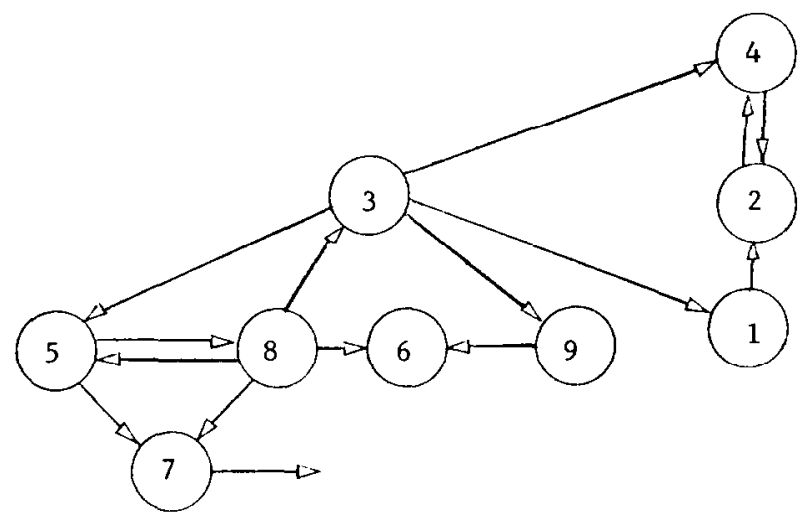

It is obvious from the diagram that $S$ is separable. Indeed, if

and

$$
S_{1}=\left\{C_{3}, C_{5}, C_{6}, C_{7}, C_{8}, C_{9}\right\}
$$

$$
S_{2}=\left\{C_{1}, C_{2}, C_{4}\right\}
$$

then $S=S_{1} \dot{\cup} S_{2}$, where $S_{1}$ receives no input from $S_{2}$. If we were to relabel the compartments at this stage, the system matrix would be in the form

$$
\left[\begin{array}{cc}
G_{11} & 0 \\
G_{21} & G_{22}
\end{array}\right]
$$

Notice this is an example of the first stage in the induction in the proof of the theorem. However, both $S_{1}$ and $S_{2}$ can be broken down. Indeed, let

$$
\begin{array}{ll}
S_{11}=\left\{C_{3}, C_{5}, C_{8}\right\}, & S_{21}=\left\{C_{1}\right\}, \\
S_{12}=\left\{C_{7}\right\}, & S_{22}=\left\{C_{2}, C_{4}\right\}, \\
S_{13}=\left\{C_{9}\right\}, & \\
S_{14}=\left\{C_{6}\right\}, &
\end{array}
$$

Then $S_{1}=S_{11} \dot{\cup} S_{12} \dot{\cup} S_{13} \dot{\cup} S_{14}$ and $S_{2}=S_{21} \dot{\cup} S_{22}$, where this partitioning has the properties listed in the theorem. Now let us relabel the compart- 
ments in $S$. The permutation for such a renumbering is

$$
\begin{aligned}
\sigma=\left(\begin{array}{lllllllll}
3 & 5 & 8 & 7 & 9 & 6 & 1 & 2 & 4 \\
1 & 2 & 3 & 4 & 5 & 6 & 7 & 8 & 9
\end{array}\right) \\
=\left(\begin{array}{llllllll}
1 & 7 & 4 & 9 & 5 & 2 & 8 & 3
\end{array}\right) \in \Sigma_{9} .
\end{aligned}
$$

Thus $C_{3}$ becomes $C_{1}, C_{5}$ becomes $C_{2}$, etc. The matrix of the system relative to the numbering may be found directly by using $\sigma$, i.e., we simply examine $\left(f_{\sigma(i), \sigma(j)}\right)$, and place the entries in their proper position. The matrix is

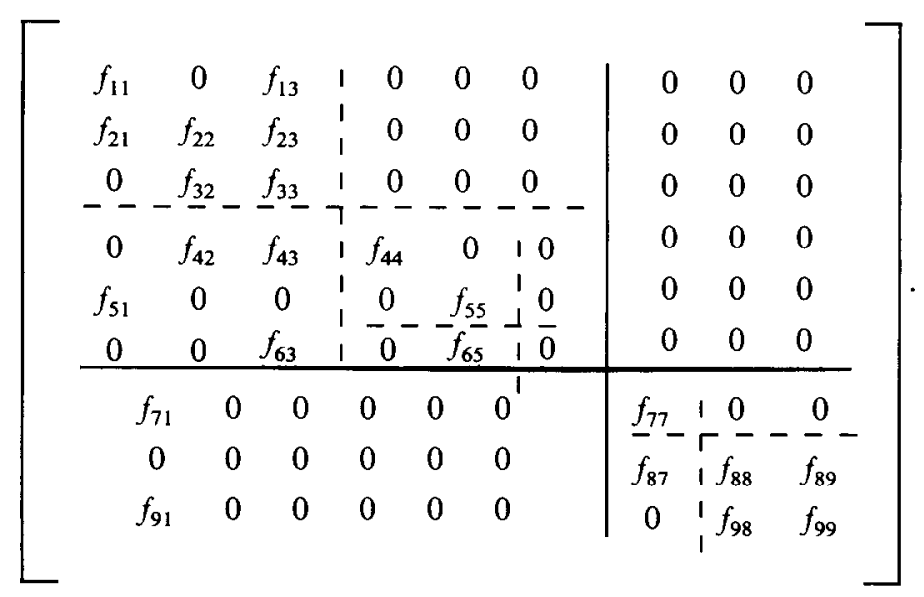

We have indicated how the system matrix can be broken down for analysis. Notice that the two (simple) traps are $S_{14}$ and $S_{22}$. Moreover, to put the matrix in the form stated in the theorem can be accomplished by $\rho=(6$ $7) \in \Sigma_{9}$. We can now analyze this system as we did in the previous example.

\section{REFERENCES}

1 D. Fife, Math. Biosci. 14, 311 (1972).

2 J. Z. Hearon, N. Y. Acad. Sci. Ann. 108, 36 (1963).

3 O. Taussky, Am. Math. Mon. 56 (10), 672 (1949). 